Article

\title{
Sustainable Rural Electrification Through Solar PV DC Microgrids-An Architecture-Based Assessment
}

\author{
Mashood Nasir ${ }^{1, *(\mathbb{D}}$, Saqib Iqbal ${ }^{2}$, Hassan A. Khan ${ }^{2}{ }^{\mathbb{D}}$, Juan C. Vasquez ${ }^{1}$ \\ and Josep M. Guerrero ${ }^{1}$ (D) \\ 1 Center for Research on Microgrids (CROM), Department of Energy Technology, Aalborg University, \\ 9220 Aalborg, Denmark; juq@et.aau.dk (J.C.V.); joz@et.aau.dk (J.M.G.) \\ 2 Department of Electrical Engineering, Lahore University of Management Sciences, Lahore 54892, Pakistan; \\ 15060005@lums.edu.pk (S.I.); hassan.khan@lums.edu.pk (H.A.K.) \\ * Correspondence: mnas@et.aau.dk; Tel.: +45-(91)-498791
}

Received: 8 October 2020; Accepted: 3 November 2020; Published: 6 November 2020

\begin{abstract}
Solar photovoltaic (PV) direct current (DC) microgrids have gained significant popularity during the last decade for low cost and sustainable rural electrification. Various system architectures have been practically deployed, however, their assessment concerning system sizing, losses, and operational efficiency is not readily available in the literature. Therefore, in this research work, a mathematical framework for the comparative analysis of various architectures of solar photovoltaic-based DC microgrids for rural applications is presented. The compared architectures mainly include (a) central generation and central storage architecture, (b) central generation and distributed storage architecture, (c) distributed generation and central storage architecture, and (d) distributed generation and distributed storage architecture. Each architecture is evaluated for losses, including distribution losses and power electronic conversion losses, for typical power delivery from source end to the load end in the custom village settings. Newton-Raphson method modified for DC power flow was used for distribution loss analysis, while power electronic converter loss modeling along with the Matlab curve-fitting tool was used for the evaluation of power electronic losses. Based upon the loss analysis, a framework for DC microgrid components (PV and battery) sizing was presented and also applied to the various architectures under consideration. The case study results show that distributed generation and distributed storage architecture with typical usage diversity of $40 \%$ is the most feasible architecture from both system sizing and operational cost perspectives and is $13 \%$ more efficient from central generation and central storage architecture for a typical village of 40 houses. The presented framework and the analysis results will be useful in selecting an optimal DC microgrid architecture for future rural electrification implementations.
\end{abstract}

Keywords: DC microgrids; DC power systems; loss analysis; Newton-Raphson; rural electrification; system sizing

\section{Introduction}

Reliable access to electricity is one of the basic indicators for the quality of life and the economic standing of any community [1,2]. The availability of electricity directly affects living standards, education facilities, modes of transportation, industrial growth, and agriculture productivity $[3,4]$. Around 800 million people worldwide have no access to electricity, and a major part (approximately $83 \%$ ) of this population belongs to rural areas [3,5]. The inhabitants of these underprivileged regions are still using fossil fuels, kerosene oil, and other raw materials to justify their basic energy needs, e.g., lighting, cooking, and heating [3,6]. Although these resources are partially fulfilling the very basic needs of the rural communities, however, these resources are not much environment friendly and also 
have adverse impacts on health [3]. Therefore, electricity access to rural communities is the need of the hour to attain the associated socioeconomic benefits.

One possible way to electrify these unelectrified villages is through the expansion of the national electricity grid and associated generation, transmission, and distribution infrastructure. However, it involves a huge cost, and developing countries with limited resource availability cannot afford such large scale expansions [3]. Alternately, distributed generation (DG) based microgrids have evolved as a reliable, affordable, and cost-effective solution for generation near to the load centers [7-9]. Direct current (DC) microgrids, in comparison to alternating current (AC) microgrids, are considered more suitable for rural applications mainly due to (a) minimal synchronization requirements, (b) higher distribution efficiency, and (c) no need of AC/DC power electronic conversion for interfacing with battery and solar photovoltaic (PV), as both are inherently DC in nature [10,11]. Additionally, the large scale market availability of highly efficient DC loads has further favored DC microgrids as a candidate choice for rural electrification [12]. Since solar photovoltaic technology offers a clean, environment friendly, and green source of energy generation, therefore, this work is primarily focused on solar PV-based DC microgrids for sustainable rural electrification. Other distributed generation resources, e.g., diesel or hybrid PV/diesenl, are not considered within the current scope of this work.

During the last decade, a large number of solar PV-based DC microgrids have been deployed for rural electrification, particularly in India, Bangladesh, and African regions [10,13-17]. One prominent project is Mera Gao Power (MGP) in Uttar Pradesh (UP), India, which was founded in 2010 and is now considered as the lowest cost commercially viable solar PV- based DC microgrid. According to the reports, MGP has the potential to connect across 5000 villages, supplying basic electricity to approximately 50,000 households and 0.3 million rural consumers [10,18]. Similarly, The Energy and Resource Institute (TERI) of India under the campaign of lighting a billion lives provided electricity to 11,000 households in 243 villages spread across six states using solar DC microgrids [10,14]. Other considerable commercial examples are Sharatipur microgrid in Bangladesh and the Worldwide Fund for Nature (WWF) supported solar DC microgrid installed in Kasese district of Uganda by joint energy and environment project (JEEP) $[16,17]$. Despite the existence of many commercial projects, power architecture for these deployments is not standardized yet, and there exist many architecture variants. For instance, the MGP and the TERI project of India are using centralized architecture, in which the PV generation and battery storage resources are placed at a central location in the village, and from that central position, power is distributed at $24 \mathrm{~V}$ to each household. On the other hand, the Sharatipur project in Bangladesh is using a distributed architecture, where PV generation and battery storage resources are distributed throughout the village across various households. Other than these practical deployments, various other architectures have been proposed in the literature with scaled down laboratory-scale implementations. For instance, Madduri et al. [19] proposed a scalable architecture using hybrid topology with PV generation at the central location, while battery storage resources are distributed across the village household. Similarly, Nasir et al. proposed a highly distributed architecture with neighborhood-level power-sharing capability implemented with decentralized control [20-22]. The previous works mainly focus on design, analysis, and optimization of individual architectures without considering the impact of architectural variations on system sizing and losses [1,20-25]. A framework for optimal planning and design of low power low voltage islanded DC microgrids for minimum upfront cost was developed in [23]. Similarly, a framework for optimal planning and design of grid-connected DC microgrid was presented in [25]. However, both of these works consider DC microgrid with central architecture and do not consider loss analysis and system sizing for other possible architectures. Distribution loss analysis for rural DC microgrids using modified Netwon-Raphson (NR) has been presented in [1], while decentralized algorithms are discussed in $[20,21,26]$. These works consider a distributed architecture of DC microgrid only and therefore do not illustrate insight on loss variations in other types of architectures. The comparison between central and distributed architectures has been performed in [27], however, this work only considers distribution losses, ignoring the impact of variations in the converter losses. Alternatively, 
in this work, we present a compact framework to comprehensively analyze all the possible architectures along with both categories of losses, including distribution and conversion losses. The framework also presents the impact of losses on system sizing in various architectures, which is also an addition to the previous works in this domain. Therefore, this work is dedicated to the detailed comparison of all the possible architecture variants of DC microgrids for rural electrification implementations, with prime focus on losses, operational efficiency, and system sizing.

Our first contribution in this research work mainly includes the classification of various DC microgrid architectures employed for rural electrification. Based upon the existing deployments and proposed implementations, various architectures of DC microgrids are identified [3,10,14-19,23]. These architectures are further classified based upon the placement of generation and storage resources and are termed as (a) central generation and central storage architecture (CGCSA), (b) central generation and distributed storage architecture (CGDSA), (c) distributed generation and central storage architecture (DGCSA), and (d) distributed generation and distributed storage architecture (DGDSA). The detailed power architectures, including component count and placement of resources for these architectures, are discussed in Section 2.

Our second contribution mainly includes the identification and the quantification of various parameters that are coupled with the architectural design and impact the operational efficiency of the microgrid system. These parameters mainly include converter count, percentage output power loading of each converter, distribution conductor size, power demand at each household, and intra-village special distribution of houses [24]. Though each of these components affects the operational efficiency of the system, their impact on system efficiency can be quantified in terms of two generic categories, i.e., (a) distribution losses and (b) power electronic conversion losses. Distribution losses are analyzed using the Newton-Raphson method modified for DC power flow as presented in [27]. However, the analysis of central and distributed architectures of solar PV DC microgrids presented in [27] considers distribution losses only, neglecting the effect of power electronic conversion losses and percentage loading of each converter at various load power demands. It has been shown in [28-30] that power electronic converters cannot be modeled as constant efficiency devices and their output is a function of their output loading. Therefore, power electronic losses significantly affect the system efficiency at variable power loading and were considered in this analysis using the curve fitting method for accurate loss assessment.

Based upon the system loss analysis, a framework to calculate the sizing of various components, including PV panel and battery packs, is presented. Therefore, our third contribution involves the formulation of the sizing framework based on the loss analysis. The presented framework is generic and is applicable for all the architecture variants, as discussed in the subsequent sections. Finally, the proposed framework is applied for the case study of a typical village having 40 houses and all the possible architectural variants to analyze the sizing requirements and the losses associated with each architecture [31].

The rest of the paper is organized as follows: Section 2 presents the discussions and the visual representation of various DC microgrid architectures employed for rural electrification applications. Section 3 presents a model and a framework for loss analysis along with the sizing considerations. Section 4 presents the case studies data for a typical village with 40 households. Section 5 presents the major findings of the applied framework for different possible architectures. These results are discussed and compared for various possible scenarios of resource placement. Based on the findings from results, a conclusion is drawn in Section 6.

\section{Commonly Used System Architecture for Rural Electrification}

The commonly used possible rural electrification architectures and their system models along with their visual presentations are discussed in this section. Various system components including the number of converters employed in each architecture are also highlighted. 


\subsection{Central Generation and Central Storage Architecture}

The most commonly employed architecture for rural electrification is the central generation and central storage architecture (CGCSA), which is structurally less complex in comparison to the other architectures. Due to centralized nature, it offers simplified controllability, enhanced reliability, and easy maintenance [32]. The schematic diagram of a village having N-number of houses electrified through CGCSA of PV DC microgrid is shown in Figure 1. In CGCSA, all generation (PV panels) and storage (batteries) resources are placed at a central location in the village, as shown in Figure 1. The power generated by PV panels is processed through a maximum power point tracking (MPPT) converter to extract the maximum energy out of the incident solar irradiance. The battery storage is used as an energy buffer to balance the irradiance variations and to supply power during non-sun hours. Generally, battery and PV panel voltage is lower, and distribution at a lower voltage may result in higher distribution losses, therefore, a boost converter is employed to increase the distribution voltage level and minimize the distribution losses.

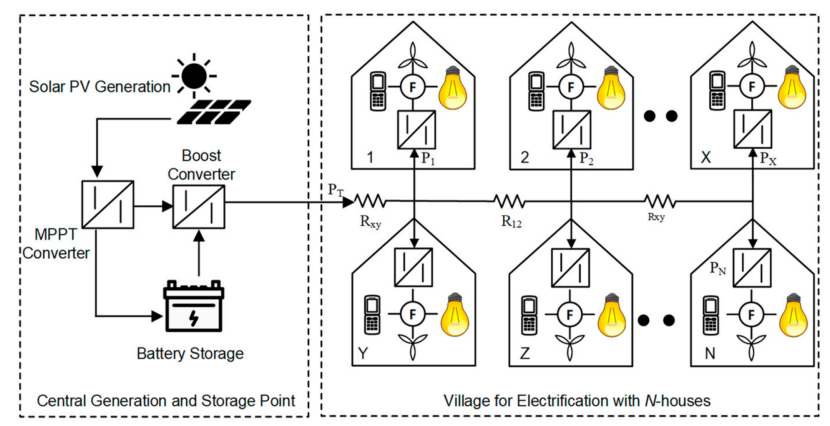

Figure 1. System diagram for centralized generation and centralized storage architecture (CGCSA).

At the consumer end, a buck converter is employed to lower down the voltage level to the household load utilization level, where load can be a mobile charger, a light bulb, or a fan (denoted by $\mathrm{F}$ in the figure). This process is exactly similar to conventional electricity transmission, distribution, and utilization, where the transformer serves the purpose of stepping up the voltage at the generation end to minimize the transmission losses; at the delivery end, a distribution transformer steps down the voltage for load utilization.

The distribution conductor is modeled in terms of distribution resistance Rxy between two arbitrary houses, $\mathrm{X}$ and $\mathrm{Y}$, where power delivery to an arbitrary household $\mathrm{X}$ is denoted by PX. Since generation, distribution, and utilization all are in DC, it is a reasonable approximation to neglect the inductance associated with the distribution conductance and consider resistance or conductance parameters only. At each household, there is a DC load that consists of lighting, fan, and mobile charging for typical off-grid electrification [3].

\subsection{Central Generation and Distributed Storage Architecture}

The central generation and distributed storage architecture (CGDSA) of the DC microgrid employed for the electrification of $N$ - house village is shown in Figure 2. In CGDSA, battery storage is distributed to individual households to reduce the losses associated with the stored energy [19]. The distribution of storage resources results in reducing the number of conversion steps and line losses in the path of energy flow from source end to load end. Moreover, the distribution of storage resources also imparts the scalability to the architecture, however, it requires relatively complex control and communication schemes among the distributed storage resources for resource balancing at a village level. The energy generated by PV panels is processed in the same manner as in the case of CGCSA. All the generated energy is distributed at a high voltage level using a DC/DC converter in the path of energy flow from a central location to each house. At the other end of the distribution line (at each house), there is another DC/DC converter, which is responsible for stepping down the voltages level to 
the household used level to supply to the load and to the battery for backup during low irradiance periods. The distribution conductor is modeled in terms of the distribution line resistance due to the DC nature of generation, storage, and loads as discussed in CGCSA.

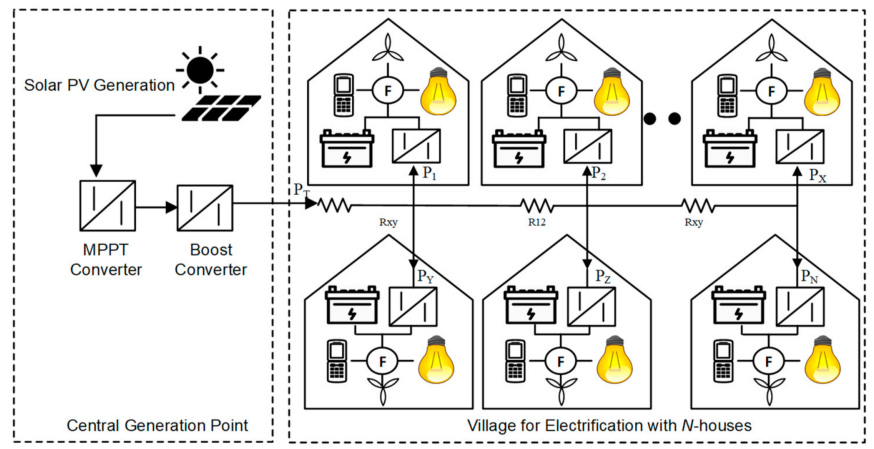

Figure 2. System diagram for centralized generation and distributed storage architecture (CGDSA).

\subsection{Distributed Generation and Central Storage Architecture}

With the development of large-scale battery storage technologies, e.g., Tesla power packs, the community level energy storage can be regarded as a viable option for both grid-connected and off-grid applications [33]. Therefore, another possible variant is distributed generation and centralized storage architecture (DGCSA), which involves the requirement of bidirectional power flow. In DGCSA, all the storage resources (battery packs) are placed at a central location in the village, while all the generation (PV panels) is distributed in various households, as shown in Figure 3. The distribution of generation resources allows the on-spot utilization of the generated energy and thereby reduces the distribution losses, however, the line that is associated with stored energy and is substantially enhanced as energy has to travel double the distance due to charging and discharging periods. The energy generated by PV panels installed at each house is processed in the same manner as discussed in previous architectures and directly supplied to the household load, while all the excess energy is distributed at a high voltage level using a bidirectional buck-boost converter. At the other end of the distribution line (central location), there is another bi-directional converter to synchronize voltage level with the battery charging and discharging requirements. The involvement of a bidirectional converter enhances the control complexity and the communication requirements for resource balancing at a village level; at the same time, shared battery resources allow surplus energy to be saved for community benefits, which may otherwise need be curtailed due to limited battery storage at individual household.

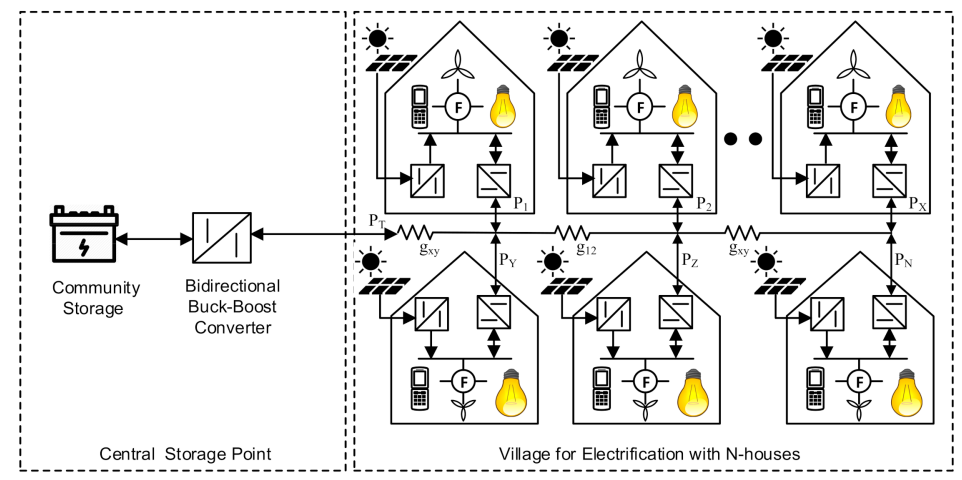

Figure 3. System diagram for distributed generation and central storage architecture (DGCSA).

\subsection{Distributed Generation and Distributed Storage Architecture}

The distributed generation and distributed storage architecture (DGDSA) of PV DC microgrid for the electrification of an $N$-houses village is shown in Figure 4. The DGDSA is structurally much 
more complex in comparison to the other architectures, as it involves a higher degree of distribution, i.e., both generation and storage resources are placed in individual households. Owing to a higher degree of distribution, the control complexity, and communication requirements are relatively higher for this kind of electrification architecture.

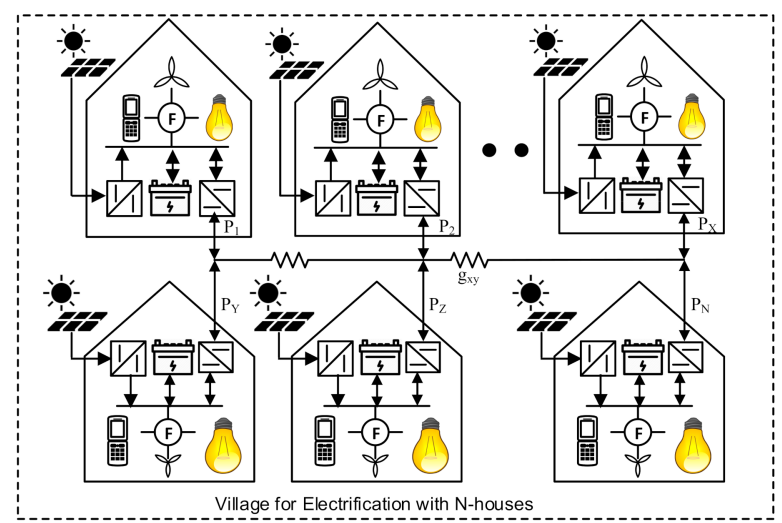

Figure 4. System diagram for distributed generation and distributed storage architecture (DGDSA).

However, at the same time, it offers multiple benefits in terms of scalability, modularity, lower distribution losses, and minimal up and down voltage conversions. Moreover, peer-to-peer power-sharing capability and power pooling for community applications are the key features of DGDSA, which otherwise are not available in other architectures $[1,3,26,27]$. The generated energy is either directly supplied to the load at a household distribution voltage level or the battery for backup during low irradiance periods. The line losses are minimal if all the houses are self-sufficient in meeting their requirements indigenously without resource sharing. However, the complexity arises when the excess energy from one household resource is supplied to any other household in the village. In power-sharing mode, the excess energy is distributed at a certain distribution voltage level (generally higher than household use level to minimize the distribution line losses) to the desired household, therefore, line losses play their part only when there is a sharing requirement. To realize this power-sharing, a DC/DC converter is employed in the path of energy flow from one household to the other household. Therefore, in this architecture, each household contains two converters, i.e., (a) unidirectional MPPT converter, and (b) bidirectional buck-boost converter, which is responsible for power-sharing among multiple neighboring houses.

The comparison of various characteristics at the component and system level among the presented architectures is shown in Table 1. 
Table 1. Comparison of various features for rural photovoltaic (PV) direct current (DC) microgrid architectures.

\begin{tabular}{|c|c|c|c|c|c|c|c|c|c|c|}
\hline \multirow{2}{*}{ Architecture } & \multicolumn{3}{|c|}{ Converter Count } & \multirow{2}{*}{$\begin{array}{c}\text { BidirectionPower } \\
\text { Flow }\end{array}$} & \multicolumn{2}{|c|}{ Scalability/Modularity } & \multirow{2}{*}{$\begin{array}{l}\text { Peer-to-PeerPower } \\
\text { Sharing }\end{array}$} & \multirow{2}{*}{$\begin{array}{l}\text { Control/Communication } \\
\text { Complexity }\end{array}$} & \multirow{2}{*}{ Maintenance } & \multirow{2}{*}{ Ref } \\
\hline & MPPT & $\mathrm{DC} / \mathrm{DC}$ & Total & & Generation & Storage & & & & \\
\hline CGCSA & 1 & $N+1$ & $N+2$ & No & No & No & No & Low & Low & {$[10,14,16]$} \\
\hline CGDSA & 1 & $N$ & $N+1$ & No & No & Yes & No & Medium & Medium & {$[19,33]$} \\
\hline DGCSA & $N$ & $N+1$ & $2 N+1$ & Yes & Yes & No & Yes & High & High & [34] \\
\hline DGDSA & $N$ & $N$ & $2 N$ & Yes & Yes & Yes & Yes & Very High & Very High & {$[1,21,35]$} \\
\hline
\end{tabular}




\section{Framework for the Estimation of Losses, and System Sizing,}

To estimate the operational efficiency of any electrification architecture, it is important to analyze key parameters including (a) load requirement at each household, (b) converter specifications including converter count and their power ratings, (c) PV panel and battery storage size, and (d) distribution conductor thickness. For operational efficiency considerations, system losses are categorized as (a) power electronics converter losses and (b) distribution losses. Other than the above-mentioned categories, there may exist other types of losses in the system, e.g., soiling, and temperature losses in PV panels and batteries charging and discharging [36-38]. However, these losses are independent of the architecture and are considered constant for architectural analysis.

Distribution losses depend upon conductor thickness, distribution voltage level, amount of distributed power, and distance among the distributed nodes. For the analysis of distribution losses in the conventional power systems, various techniques are employed, e.g., Gauss-Seidel, Newton-Raphson, decoupled power flow, however, these techniques are designed for AC power systems and need to be modified for the loss analysis in DC distributed systems [27]. The framework discussed below employs the Newton-Raphson method modified for DC power flow analysis for distribution loss calculation in each architecture [27]. In each of the architectures, DC/DC converters are used either to perform MPPT operation or step-up and step-down voltage conversion. However, power processing at each converter results in losses that can be modeled as constant losses and variable losses collectively termed as power electronic losses. These losses are dependent upon the characteristics of active and passive switches along with the filter elements in each converter, however, they can be modeled as a function of output power loading [12]. Based on the loss analysis, a mathematical framework to estimate operational efficiency and sizing is presented in the following subsections.

\subsection{Distribution Loss Analysis Using Newton-Raphson Modified for DC Power Flow}

In any architecture, distribution losses $P_{d}(t)$ at any time $t$ are a function of (a) number of houses in the architecture $N$, (b) distribution voltage level $V^{d}$, (c) power demand at each household $P_{x}$, (d) distance between houses and associated length and thickness of the distribution conductor. To quantify distribution losses, an $N$-house village is modeled as a combination of interconnection resistances $R_{x y}$ of the laid distribution conductors between any two arbitrary houses, $x$ and $y$, as shown in Figures 1-4. As discussed earlier, since generation, distribution, and utilization all involve direct current, therefore, the inductance of the distribution conductor can be fairly neglected while modeling the distribution network. The distribution structure can be radial, ring, or interconnected mesh network, and, therefore, conductance matrix $G$ (just like bus admittance matrix in the case of AC power flow analysis) can be formulated depending upon the spatial distribution between house and the associated length of the distribution conductor, as given by Equation (1):

$$
G=\left[\begin{array}{cccccc}
G_{11} & G_{12} & \ldots & G_{x y} & \ldots & G_{1 N} \\
G_{21} & G_{22} & \ldots & G_{x y} & \ldots & G_{2 N} \\
\vdots & \vdots & \ddots & \vdots \\
G_{N 1} & G_{N 2} & \ldots & G_{x y} & \ldots & G_{N N}
\end{array}\right] ; G \in \mathfrak{R}^{N \times N} ; \forall x \in[1, N] ; \forall y \in[1, N]
$$

where $G$ is the conductance matrix of the distribution network, and its order is of the order $N \times N$, and $G_{x y}$ 's are the individual entries of the conductance matrix. Similar to the bus admittance matrix in conventional AC power systems, diagonal and off-diagonal elements of the conductance matrix can be calculated by Equation (2):

$$
G_{x y}=\left\{\begin{array}{cc}
\sum_{y=1}^{N} \frac{1}{R_{x y}} & ; \forall \mathrm{x}=y \\
-\frac{1}{R_{x y}} & ; \forall \mathrm{x} \neq \mathrm{y}
\end{array}\right\} ; \forall x \in[1, N] ; \forall y \in[1, N]
$$


For each architecture, as shown in Figures $1-4$, the total power delivery $P_{T}$ from generation resources to the household loads can be arranged in the form of a scheduling matrix $P_{\text {sch }}$ in terms of power delivery at each arbitrary household $P_{X}$ as shown by Equation (3):

$$
P_{\text {sch }}=\left[\begin{array}{lllll}
P_{1} P_{2} \ldots \ldots & \ldots & \ldots & \ldots & P_{N}
\end{array}\right] ; P_{s c h} \in \mathfrak{R}^{1 \times N} ; \forall x \in[1, N]
$$

Based on the conductance matrix model of the village, the calculated power matrix $P_{c a l}$ is given by (4) in terms of its entries calculated by (5):

$$
\begin{gathered}
P_{\text {cal }}=\left[P_{1}^{*} P_{2}^{*} \ldots P_{x}^{*} \ldots P_{N}^{*}\right] ; P_{c a l} \in \mathfrak{R}^{1 \times N} ; \forall x \in[1, N] \\
P_{x}^{*}=\sum_{y=1}^{N} V_{x}^{d} V_{y}^{d} G_{x y} ; \forall x \in[1, N]
\end{gathered}
$$

where $V_{x}{ }^{d}$ and $V_{y}{ }^{d}$ are the reference voltage levels at the distribution interface of arbitrary households $x$ and $y$. Considering one of the buses as the reference bus, the difference between scheduled and calculated power matrices $\Delta P$ can be iteratively minimized to find the value of voltage difference matrix $\Delta P$ at each household interface using a Jacobian matrix, as given by Equations (6) and (7) [38]:

$$
\begin{aligned}
& \Delta V=J^{-1} \Delta P ; \Delta V \in \mathfrak{R}^{1 \times N-1} ; J \in \mathfrak{R}^{N-1 \times N-1} ; \Delta P \in \mathfrak{R}^{1 \times N-1} \\
& {\left[\begin{array}{c}
\Delta V_{2}^{(k)} \\
: \\
\Delta V_{x}^{(k)} \\
\vdots \\
\Delta V_{N}^{(k)}
\end{array}\right]=\left[\begin{array}{ccccc}
\frac{\partial P_{2}^{(k)}}{\partial V_{2}} & \cdots & \frac{\partial P_{2}^{(k)}}{\partial V_{x}} & \cdots & \frac{\partial P_{2}^{(k)}}{\partial V_{N}} \\
\vdots & & : \\
\frac{\partial P_{X}^{(k)}}{\partial V_{2}} & \ddots & : \\
\vdots & \ddots & \vdots \\
\frac{\partial P_{N}^{(k)}}{\partial V_{2}} & & \frac{\partial P_{2}^{(k)}}{\partial V_{N}}
\end{array}\right]^{-1}\left[\begin{array}{c}
\Delta P_{2}^{(k)} \\
\vdots \\
\Delta P_{x}^{(k)} \\
\vdots \\
\Delta P_{N}^{(k)}
\end{array}\right]}
\end{aligned}
$$

Voltage vectors are updated for each iteration, and the iterative process continues for $k$ iterations until the difference in power for each household falls below the predefined tolerance level. After the convergence, the updated voltage matrix is used to calculate the distribution losses, as shown by Equation (8):

$$
P_{d}=\sum_{x=1}^{N} \sum_{y=1}^{N} G_{x y}\left[V_{x}^{d}\left(V_{x}^{d}-V_{y}^{d}\right)+V_{y}^{d}\left(V_{y}^{d}-V_{x}^{d}\right)\right]
$$

where $V_{x}{ }^{d}$ and $V_{y}{ }^{d}$ are the voltages at the distribution interface of arbitrary houses, $x$ and $y$, after convergence.

\subsection{Power Electronic Conversion Loss Analysis}

The power electronic conversion losses of the microgrid architecture depend upon the number of conversion stages encountered in the path of power flow from the source end to the load end. These converters mainly include (a) an MPPT converter that is responsible for optimal PV integration and battery charging, (b) a step-up converter for power distribution at a relatively higher voltage, and (c) the load converter responsible for stepping down the voltage to the utilization level. The power electronic loss induced by each converter mainly consists of switching losses, conduction losses, and leakage losses [28]. The conversion efficiency of power electronic converter can be modeled as a non-linear function of its output loading, as given by Equation (9) $[12,28]$ :

$$
\eta_{c}=\sum_{i=0}^{K} k_{i}\left(\frac{P}{P_{R}}\right)^{i}
$$


where $P_{R}$ is the rated power of the converter, $P_{o}$ is the output power drawn from the converter at the given loading scenario, and $k_{i}$ 's are the coefficients of energy conversion efficiency ranging from 0 to $K$. In general, for the simplified analysis, higher-order terms are neglected, and $K$ is limited to the lower order, i.e., $K=2$, which results in the simplification of Equation (9) in terms of Equation (10), as shown below [27]:

$$
\eta_{c}=k+k_{1}\left(\frac{P}{P_{R}}\right)^{1}+k_{2}\left(\frac{P}{P_{R}}\right)^{2}
$$

However, simplification to lower-order coefficients may compromise the accuracy of analysis, and, therefore, higher-order coefficients need to be considered. Using the manufacturer's datasheet parameters, a curve-fitting based method can be employed for the extraction of higher-order parameters of (9) for the accurate analysis of conversion efficiency and power electronic losses. The power electronic conversion losses incurred during $i^{\text {th }}$ conversion stage $P_{c}{ }^{i}$ can therefore be calculated using Equation (11):

$$
P_{c}=\frac{1-\sum_{i=0}^{K} k_{i}\left(\frac{P}{P_{R}}\right)^{i}}{\sum_{i=0}^{K} k_{i}\left(\frac{P}{P_{R}}\right)^{i}}
$$

\subsection{Power Flow Diagrams and Village Scale PV Sizing Estimation}

In order to estimate the total system power losses including conversion and distribution losses, it is necessary to visualize how power flows from the source end to the load end. Power flow diagrams presented below help to visualize the losses encountered in the path of power flow. Based on various components defined in the path of power flow, a compact formulation is formed for determining the operational efficiency of each architecture. Since the sizing of the system is directly dependent upon the system losses, power flow diagrams also give a fair idea of the system sizing calculation detailed in the sub-sections.

\subsubsection{Power Flow Diagram and PV Sizing Estimation for CGCSA}

The power flow diagram for CGCSA is shown in Figure 5. From the architecture (presented in Figure 1), it can be seen that the total power demanded by all the household loads $P_{L}(t)$ at any time $t$ is given by Equation (12):

$$
P_{L}(t)=\sum_{X=0}^{N} P_{X}^{\prime}(t)
$$

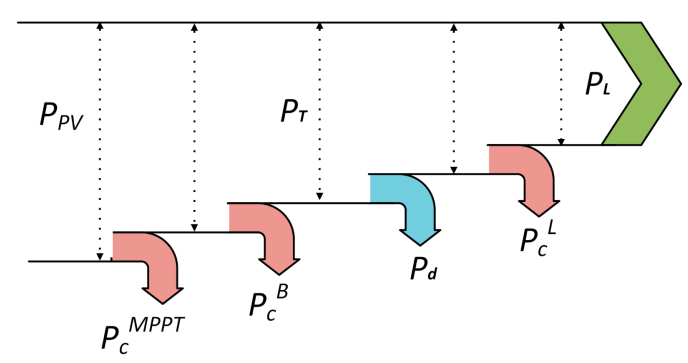

Figure 5. Power flow diagram for central generation and central storage architecture.

The load power is fulfilled by the load converters installed at each household; therefore, based on output power loading and efficiency characteristics, input power needed at each household interface can be calculated using Equation (13):

$$
P_{X}(t)=P_{X}^{\prime}(t)+P_{c}^{L}
$$


where $P_{c}{ }^{L}$ corresponds to the power electronic conversion losses across the household load converter and can be calculated using Equation (11) and load converter efficiency coefficients $k_{i}{ }^{L}$ as given by Equation (14):

$$
P_{c}^{L}=\frac{1-\sum_{i=0}^{K} k_{i}^{L}\left(\frac{P_{X}^{\prime}(t)}{P_{R}^{L}}\right)^{i}}{\sum_{i=0}^{K} k_{i}^{L}\left(\frac{P_{X}^{\prime}(t)}{P_{R}^{L}}\right)^{i}}
$$

Once the power required at each household node is calculated, distribution losses across the distribution network can be found by scheduling the known amount of power at each household in Equation (3) and following the NR method modified for DC power flow, as mentioned above using Equations (4)-(8). Therefore, following the power flow diagram, the total power required at the input of distribution network $P_{T}$ can be calculated using Equation (15):

$$
P_{T}(t)=P_{d}(t)+\sum_{x=1}^{N} P_{x}
$$

Similarly, going across the power flow diagram and solving for boost converter losses $P_{C}{ }^{B}$ and MPPT converter losses $P_{C}{ }^{M P P T}$ using Equation (11) yields us the total power required from PV panel at any time $t$, as given by Equation (16).

$$
P_{P V}(t)=P_{T}(t)+P_{C}^{B}(t)+P_{C}^{M P P T}(\mathfrak{t})
$$

If the incident irradiance in terms of average peak sun hours (PSH) is known for the location under consideration, this energy can be used for the calculation of PV panel sizing $S_{P V}\left(W_{p}\right)$ required to fulfill the given amount of load, as shown by Equation (17):

$$
S_{P V}\left(W_{p}\right)=\frac{1}{P S H}\left[\sum_{t=1}^{T} P_{P V}(t)\right]
$$

where $T$ is the total number of hours in a day, and $P_{P V}(t)$ is the total amount of time-varying power required for the load fulfillment. Therefore, when routing across the proposed power flow diagrams, not only the system losses but also the sizing of the system for a given architecture under consideration can be calculated.

\subsubsection{Power Flow Diagram and PV Sizing Estimation for CGDSA}

In the case of CGCSA, the generated power is stored locally and is distributed only when there is a load requirement; therefore, power distribution losses are independent of the incident irradiance and PV generation profile. On the contrary, in CGDSA, due to the unavailability of the local battery, all the generated power is distributed to household batteries in the duration of sun hours, when high irradiance is available. Since distribution losses increase in a quadratic fashion with the amount of power to be distributed, this architecture incurs higher distribution losses in PV generation hours. This quadratic increase in distribution losses is shown via a large length arrow in Figure 6. 


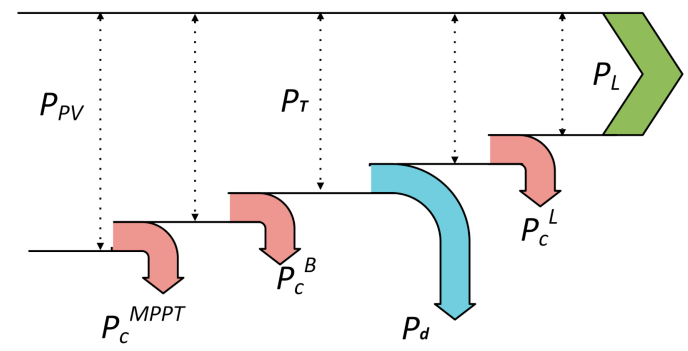

Figure 6. Power flow diagram for central generation and distributed storage architecture.

In order to estimate the PV sizing and system losses in CGDSA, the instantaneous power produced by PV panel $P_{P V}(t)$ needs to be represented in terms of its ambient conditions, including its cell temperature $T_{\mathcal{c}}$, ambient temperature $T_{a m b}$, incident irradiance $I\left(\mathrm{~W} / \mathrm{m}^{2}\right)$, and temperature compensated incident irradiance $I_{t c}\left(\mathrm{~W} / \mathrm{m}^{2}\right)$, as given by Equation (18) [25].

$$
P_{P V}(t)=A \cdot \eta_{m} \cdot I_{t c}(t)
$$

where $A$ is the area of the PV panels, and $\eta \mathrm{m}$ is the module conversion efficiency. The temperature-compensated irradiance depends upon $I(t)$ and $T_{c}$ and is given by Equation (19) [39]:

$$
\begin{gathered}
T_{c}(t)=T_{a m b}(t)+(0.01875 * I(t)) \\
I_{t c}(t)=\left(1-\left(T_{c}-25\right) * 0.0045\right) I(t)
\end{gathered}
$$

The initial estimate of PV sizing is taken from total load demand at individual households, however, size is updated after each loss component, as shown in the power flow diagram. Therefore, losses across the load/battery converter $P_{c}{ }^{L}$ are a function of run-time generated PV power and are processed by each household converter in terms of load converter efficiency coefficients $k_{i}{ }^{L}$, as given by Equation (21):

$$
P_{c}^{L} \approx \frac{1-\sum_{i=0}^{K} k_{i}^{L}\left(\frac{P_{P V}(t)}{P_{R}^{L}}\right)^{i}}{\sum_{i=0}^{K} k_{i}^{L}\left(\frac{P_{P V}(t)}{P_{R}^{L}}\right)^{i}}
$$

At this stage, distribution losses are calculated by scheduling power at each household and applying the NR method modified DC power flow as mentioned above. The overall distribution losses depend upon the amount of energy to be stored in each household and can be optimized using optimal charge/discharge algorithms based on each household requirements and forecasted load profile. However, in the current scope of the work, we assumed equal power storage at each household.

$$
P_{T}(t)=\sum_{X=1}^{N} P_{x}^{\prime}+P_{d}(t)+P_{c}^{L}(t)
$$

Similarly, power loss across the boost and the MPPT converters are also calculated using Equation (11), and overall PV sizing may be determined using Equation (23), as shown below:

$$
S_{P V}\left(W_{p}\right)=\frac{1}{P S H}\left[\sum_{t=1}^{24}\left(P_{T}(t)+P_{c}^{M P P T}(t)+P_{c}^{B}(t)\right)\right]
$$

\subsubsection{Power Flow Diagram and PV Sizing Estimation for DGCSA}

In the case of DGCSA, part of the generated PV power is consumed locally by the household loads, while the remaining power is distributed to the central battery. Therefore, DGCSA has to encounter 
distribution losses during charging as well as discharging of the battery, as shown in Figure 7. In order to estimate the overall system losses and PV sizing, charging and discharging instances of the battery need to be segregated. At any given instant of time $t$, each household is either supplying power for battery charging, incurring charging distribution power loss $P_{d}{ }^{c h}$, or it is demanding power from the central battery, incurring discharging distribution power $\operatorname{loss} P_{d}{ }^{d i s}$. Therefore, if the power generation of the household is higher than its local demand, it aids in central battery charging, otherwise, it demands power, resulting in the discharge of the central battery.

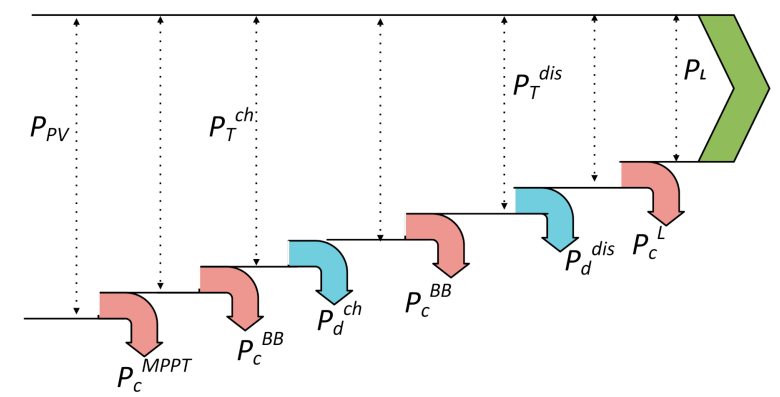

Figure 7. Power flow diagram for distributed generation and central storage architecture.

The total power for the discharging stage $P_{T}$ dis is calculated by the sum of individual discharging requirements by each household $P_{X}{ }^{d i s}(t)$ as specified by Equations (24) and (25):

$$
\begin{gathered}
P_{X}^{d i s}(t)=\left\{\begin{array}{l}
\left|P_{P V}(t)-P_{c}^{M P P T}(t)-P_{X}^{\prime}(t)\right|+P_{c}^{b o o s t} ; \forall P_{P V}(t)-P_{c}^{M P P T}(t)<P_{X}^{\prime}(t) \\
0 \forall P_{P V}(t)-P_{c}^{M P P T}(t) \geq P_{X}^{\prime}(t)
\end{array}\right. \\
P_{T}^{d i s}(t)=\sum_{X=1}^{N} P_{X}^{d i s}(t)
\end{gathered}
$$

Here, $P_{x}{ }^{\text {dis }}$ can be used to calculate the distribution losses $P_{d}$ dis during discharging state using the NR method modified for DC power flow using Equations (4)-(8). Similarly, the power for the charging stage $P_{T}{ }^{c h}(t)$ can be calculated by the summation of charging contributions by the individual households $P_{X}{ }^{c h}(t)$, as specified by using Equations (26) and (27):

$$
\begin{aligned}
& P_{X}^{c h}(t)=\left\{\begin{array}{l}
0 \quad ; \forall P_{P V}(t)-P_{c}^{M P P T}(t)<P_{x}^{\prime}(t) \\
P_{P V}(t)-P_{c}^{M P P T}(t)-P_{x}^{\prime}(t)+P_{c}^{b o o s t} ; \forall P_{P V}(t)-P_{c}^{M P P T}(t) \geq P_{x}^{\prime}(t)
\end{array}\right. \\
& P_{T}^{c h}(t)=\sum_{x=1}^{N} P_{x}^{c h}(t)
\end{aligned}
$$

Here, $P_{x}{ }^{\text {ch }}$ can be used to calculate the distribution losses $P_{d}^{c h}$ during the charging state using the NR method modified for DC power flow as defined above. The converter losses, including MPPT conversion losses for each converter $P_{c}{ }^{M P P T}$, buck-boost converter losses $P_{c}{ }^{B B}$, and load converter losses $P_{c}{ }^{L}$, can be evaluated using Equation (11) as discussed in the above architectures. Following the diagram of power flow, village-scale PV sizing for DGCSA can be calculated using Equation (28):

$$
S_{P V}\left(W_{p}\right)=\frac{1}{P S H}\left[\sum_{t=1}^{24}\left(\sum_{X=1}^{N}\left(P_{X}^{\prime}(t)+P_{c}^{M P P T}(t)+P_{c}^{L}(t)\right)+P_{c}^{B B}(t)+P_{d}^{d i s}(t)+P_{c}^{B B}(t)+P_{d}^{c h}(t)\right)\right]
$$

\subsubsection{Power Flow Diagram and PV Sizing Estimation for DGCSA}

In the case of DGDSA, if every household is self-sufficient, i.e., it can fulfill its household load demand using indigenous PV generation and battery resources, distribution losses become zero, 
and only converter losses incur in the path of power flow from the source end to the load end. However, as described earlier, DGDSA may have the capability to share power among the neighboring households, therefore, distribution losses become a function of power-sharing among households. With the percentage increase in power-sharing, distribution losses increase as shown in Figure 8 below. Not only the distribution losses but also the converter losses become a function of power-sharing among households as shown by the power flow diagram of DGDSA.

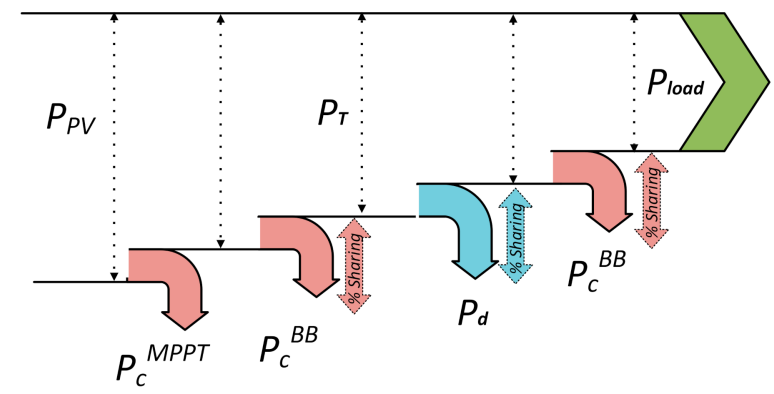

Figure 8. Power flow diagram for distributed generation and distributed storage architecture.

Firstly considering the scenario where every household is self-sufficient, the load demand is fulfilled by either the PV generation or the battery storage. Since battery voltage is kept the same as load voltage, all the generated power is processed through the common battery/load bus as shown in Figure 4. The MPPT converter losses become $P_{C}{ }^{M P P T}$ becomes a function of incident irradiance and ambient conditions as explained by Equations (18)-(20), and overall village scale size can be determined using a simple relationship defined by Equation (29):

$$
S_{P V}\left(W_{p}\right)=\frac{1}{P S H}\left[\sum_{t=1}^{T}\left(P_{X}^{\prime}(t)+P_{c}^{M P P T}(t)\right)\right]
$$

However, with the increase in power-sharing among neighboring households, distribution and conversion losses become evident and need to be calculated for the overall PV size determination. Therefore, following the power flow diagram, if any of the houses are not operating in self-sufficient mode, either supply power or demand power from the DC bus link through, which is connected with the neighboring households. In either case, distribution losses may be calculated using the information of power processed by buck-boost converters and distribution losses. For a given percentage of power-sharing $S$, the conversion losses across the buck-boost converter at the distribution interface of the supplying and receiving household can be determined using Equation (30):

$$
P_{c}^{B B}=\frac{1-\sum_{i=0}^{K} k_{i}^{B B}\left(\frac{S P_{X}^{\prime}(t)}{P_{R}^{B B}}\right)^{i}}{\sum_{i=0}^{K} k_{i}^{B B}\left(\frac{S P_{X}^{\prime}(t)}{P_{R}^{B B}}\right)^{i}}
$$

The distribution losses $P_{d}$ across the power-sharing network can be calculated using the scheduled load power matrix in terms of power-sharing coefficient $S$ and load demand at each household $P_{X^{\prime}}$, as defined by Equation (31), and applying the procedure defined by Equations (4)-(8):

$$
P_{\text {sch }}=\left[ \pm S P_{1}^{\prime} \pm S P_{2}^{\prime} \ldots \ldots \pm S P_{X}^{\prime} \ldots \ldots \pm S P_{N}^{\prime}\right] ; P_{\text {sch }} \in \mathfrak{R}^{1 \times N} ; \forall x \in[1, N]
$$


where positive $S$ corresponds to the power supplying household in the network, and negative $S$ corresponds to the power-consuming household. Once conversion and distribution losses are determined, the overall village-level PV sizing can be determined using Equation (32):

$$
S_{P V}\left(W_{p}\right)=\frac{1}{P S H}\left[\sum_{t=1}^{24}\left(\sum_{X=1}^{N}\left(P_{X}^{\prime}(t)+P_{c}^{M P P T}(t)\right)+P_{c}^{B B}(t)+P_{d}(t)+P_{c}^{B B}(t)\right)\right]
$$

\subsection{Village Scale Battery Sizing}

Since power generation from solar PV is intermittent and subject to variation in weather and ambient conditions of temperature and incident irradiance, therefore, the battery storage system not only provides the buffer for variations but also provides power during the non-available sun hours. In order to calculate the size of the battery, various parameters including battery technology (lead-acid, Li-ion, Ni-Cd, etc.), price, energy and volume density, and life cycle considerations are taken into account. Moreover, battery efficiency and depth of discharge are other key parameters that need to be considered for practical implementations [40]. Limiting the depth of discharge to higher values generally enhances the lifetime of the battery as well as reduces the maintenance cost [40]. Taking all these factors into consideration and the availability of the village scale PV size, a first-order cost calculation model is determined for the village-scale battery sizing $S_{B}(\mathrm{Wh})$. It should be mentioned that, once the solar PV size is determined, the battery sizing is independent of the architecture, and the first-order cost model presented by (33) is valid for all the architectures under consideration. The battery system must be capable of storing and supplying the total energy when the sun is not available. Along with that, it must also store extra energy dissipated during the charge/discharge cycle. Moreover, to extend the battery life, batteries are generally oversized such that there is a limit on minimum discharging state $S O C_{\min }(\%)$. The overall battery sizing for each of the architecture is given by Equation (33) [35]:

$$
S_{B}(W h)=\left[\left(1+S O C_{\min }\right)\left(\frac{N}{\eta_{B}}\right)\left(\frac{T-P S H}{T}\right) \sum_{t=1}^{T}\left(P_{P V}(t)\right)\right]
$$

where $\eta_{B}$ is the battery charge-discharge cycle efficiency, $T$ is the total number of hours in the day, PSH is the estimated average peak sunlight hours, and $P_{P V}(t)$ is the time-varying power required to fulfill the load as determined in the above sections.

\section{Case Study Parameters}

For the purpose of comparative analysis, parameters of a typical linear village generally found in the Asian rural settlements are considered [24]. Various parameters of the case studies are illustrated in Table 2. Along with constant parameters, time-varying parameters including incident solar irradiance [41], time-varying rural household load profile [42] and converter efficiency vs. loading curves for boost, MPPT, and buck-boost converter $[28,30]$ are also illustrated below in Figure 9a,b.

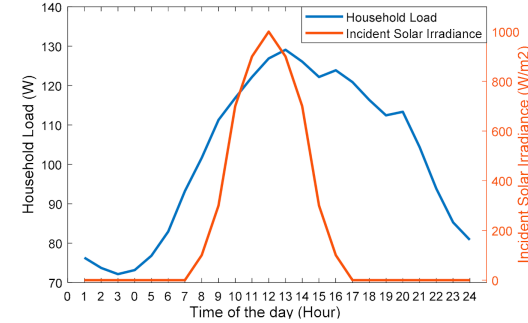

(a)

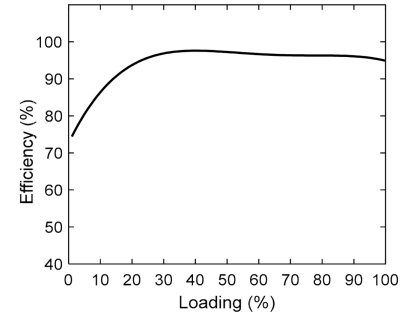

(b)

Figure 9. (a) Time-varying profile of the household load (left y-axis) and incident irradiance (right y-axis) (b) converter efficiency vs. percentage loading curve. 
Table 2. Case study parameters.

\begin{tabular}{cccc}
\hline Sr. & Symbol & Parameter Description & Value \\
\hline 1 & $N$ & Total number of houses in the village & 40 \\
\hline 2 & $R_{x y}$ & Distribution conductor resistance between two houses & $10-40 \mathrm{~m} \Omega$ \\
\hline 3 & AWG & Distribution conductor size (American Wire Gauge) & AWG 2, AWG 8 \\
\hline 4 & $P_{X}$ & Load power demand at each household X & $30-130 \mathrm{~W}$ \\
\hline 5 & $P S H$ & Average peak sunlight hour & $5.5 \mathrm{hr} /$ day \\
\hline 6 & $S O C_{m i n}$ & Minimum allowable battery state of charge & $40 \%$ \\
\hline 7 & $T$ & Operational hours in a day & $24 \mathrm{~h}$ \\
\hline 8 & $V^{d}$ & Distribution voltage level & $120 \mathrm{~V}$ \\
\hline 9 & $\mathrm{~V}^{\mathrm{B}}$ & Load bus/ battery voltage & $12-24 \mathrm{~V}$ \\
\hline 10 & $\eta_{B}$ & Battery charge-discharge cycle efficiency & $95 \%$ \\
\hline 11 & $\eta_{\mathrm{m}}$ & PV module efficiency & $18 \%$ \\
\hline 12 & $\mathrm{k}_{0}$ & Zero-order converter efficiency curve fitting constant & 72.6637 \\
\hline 13 & $\mathrm{k}_{1}$ & First-order converter efficiency curve fitting constant & 1.7731 \\
\hline 14 & $\mathrm{k}_{2}$ & Second-order efficiency curve fitting constant & 0.0448 \\
\hline 15 & $\mathrm{k}_{3}$ & Third-order converter efficiency curve fitting constant & $4.739 \times 10^{-4}$ \\
\hline 16 & $\mathrm{~K}_{4}$ & Fourth-order efficiency curve fitting constant & $-1.18 \times 10^{-5}$ \\
\hline & & &
\end{tabular}

\section{Results and Discussions}

For the comparative analysis of the presented architectures, distribution losses (W), conversions losses $(\mathrm{W}), \mathrm{PV}$ sizing $\left(\mathrm{W}_{\mathrm{p}}\right)$, and battery sizing $(\mathrm{Wh})$ are calculated using the proposed framework and the case study parameters. The findings in the form of graphical results are illustrated below. Further, it has been observed that CGCSA and DGDSA without sharing among households outperform compared to CGDSA and DGCSA, therefore, various sharing scenarios of DGDSA are compared with the base scenario of CGCSA for the comparative evaluation.

\subsection{Distribution and Power Electronic Conversion Loss Analysis}

Figure 10a,b show the power electronic conversion losses and the distribution losses across the four architectures, respectively. From Figure 10a, it is evident that power electronic conversion losses follow the same trend as those of input irradiance, as all the input power has to be processed by power electronic converters. It can also be seen in Figure 10a that conversion losses incurred in DGDSA are minimal, while DGCSA experiences a very high degree of conversion losses.

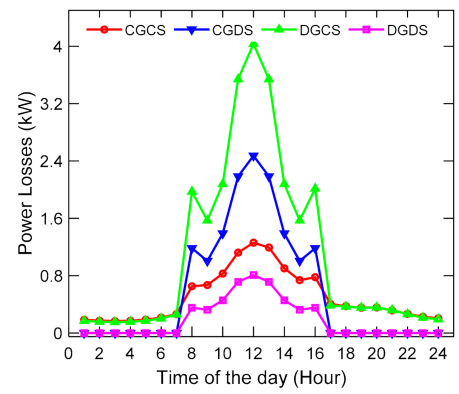

(a)

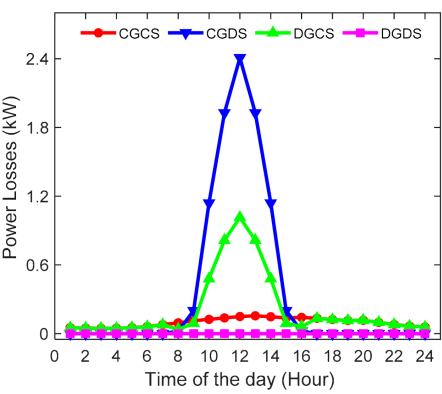

(b)

Figure 10. (a) Time-varying power electronic conversion losses of the four architectures (b) time-varying distribution losses of the four architectures. 
This is primarily due to the fact that the number of power electronic conversion stages in DGCSA is relatively higher in comparison to any other architecture, as evident from Table 1. Moreover, the power electronic losses in CGCSA (the most commonly deployed architecture) are also higher compared to the DGDSA if there is no sharing among the households. Therefore, for a general comparison, CGCSA and DGDSA outperform the other two architectures under consideration.

A similar trend can also be observed in Figure $10 \mathrm{~b}$ presenting the comparative distribution losses of the four architectures. However, here, it can be observed that, contrary to the previous case, rather DGCSA, CGCSA incurs the highest distribution losses. This is primarily due to the fact that, due to the unavailability of the central battery, all the generated energy has to be distributed among households containing distributed batteries. During the daytime, i.e., when high irradiance from the sun is available, central PV panels generate a higher amount of energy. Consequently, distribution losses increase in a quadratic proportion with the amount of the distributed power. On the contrary, in DGCSA, there are more charging and discharging stages available (refer to Figure 7), and the architecture is capable of distributing power in non-sunlight hours. Consequently, it incurs lower distribution losses. Further, it may be noted that CGCSA has minimal distribution losses, and DGDSA has zero distribution losses as long as each household is self-sufficient and there is no energy transfer among the neighboring households.

From the overall analysis of Figure 10, it can be concluded that DGDSA is the most efficient architecture concerning distribution and conversion losses, however, the scenario changes when we consider usage diversity and power-sharing among multiple neighboring households. Figure 11 shows the comparison between the most commonly employed CGCSA and various levels of power-sharing in DGDSA evaluating power electronic conversion and distribution losses. The power-sharing among the neighboring houses is increased such that, for a given percentage of power-sharing, half of the houses supply a given percentage of their peak load, and the remaining half of houses receive that amount of power. Therefore, DGDSA is evaluated and compared with CGCSA considering variable power-sharing scenarios ranging from $20 \%$ to $100 \%$. Figure 11 a compares the power electronic conversion losses, while Figure $11 \mathrm{~b}$ presents the comparison of distribution losses between CGCSA and various levels of power-sharing in DGDSA.

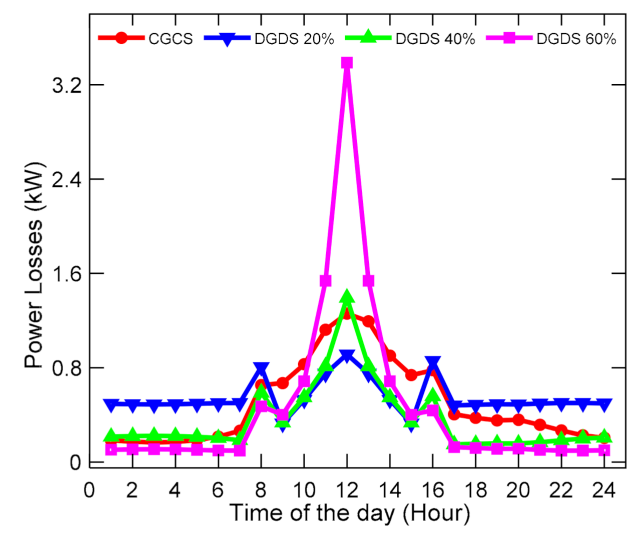

(a)

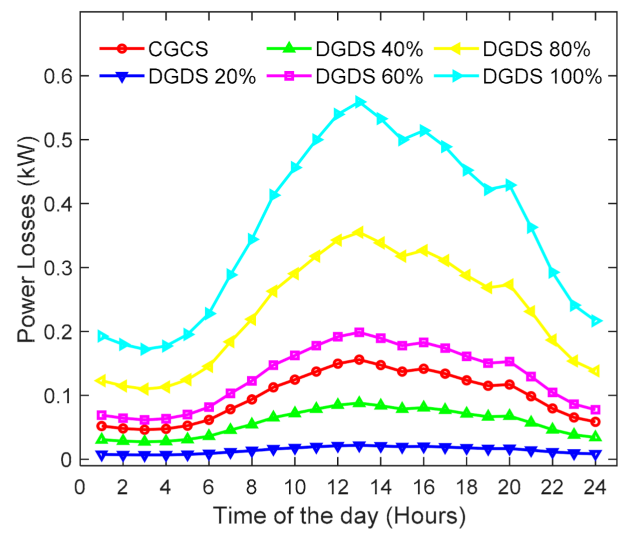

(b)

Figure 11. (a) Time-varying power electronic conversion losses of CGCSA vs. DGDSA at variable power-sharing (b) time-varying distribution losses of CGCSA vs. DGDSA at variable power-sharing.

It may be observed from Figure 11a that at 40\% power-sharing, power electronic conversion losses in DGDSA become comparable with CGCS, and for all other higher levels of power-sharing, DGDSA becomes inefficient in comparison to CGCSA, as shown in the figure. A very interesting trend can be observed here that power electronic conversion losses do not vary linearly with the percentage power-sharing. 
For instance, in Figure 11a, it may be observed that power electronic conversion losses at 20\% power-sharing are much higher compared to DGDSA with $40 \%$ power-sharing. This is primarily due to the fact that the conversion loading vs. efficiency curve is not linear, and a similar trend is also evident from Figure 9b. The converters exhibit lower efficiency at the lower loading levels, and as a result, conversion losses are higher at a lower percentage of power-sharing, as shown in Figure 11. From Figure 11b, it may be observed that, for a lower percentage of power-sharing, DGDSA exhibits lower distribution losses, however, with the increase in percentage power-sharing, conversion losses increase significantly. For power-sharing percentages higher than $40 \%$, CGCSA is more efficient in comparison to DGDSA, and distribution loss increases quadratically with the increase in percentage power-sharing. Therefore, from the overall analysis of Figure 11, it may be concluded that DGDSA with a moderate level of power-sharing (neither too low nor too high, as power electronic losses become significant at lower power-sharing) is efficient in comparison to CGCSA, however, for higher usage diversity and associated higher power-sharing percentages, CGCSA is preferable concerning distribution and conversion losses.

Lastly, a comparison between energy losses over a full-day operation $(\mathrm{kWh})$ is shown in Figure 12. Figure 12a shows the energy loss comparison between four architectures, while Figure 12b shows the comparison between commonly deployed CGCSA and DGDSA with various power-sharing provisions.

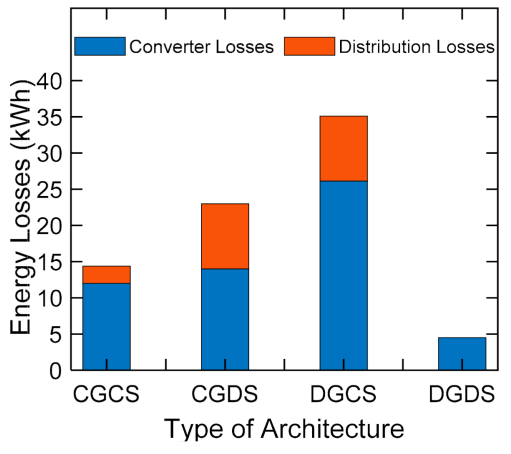

(a)

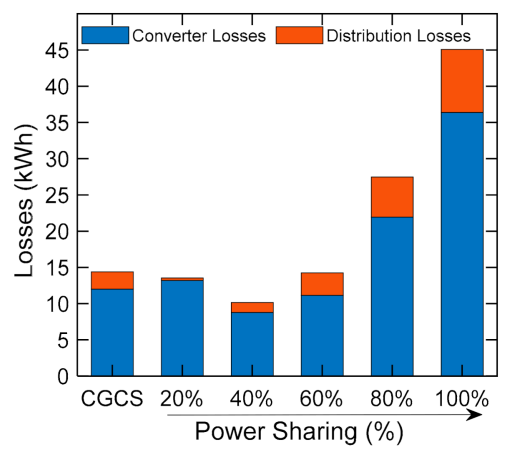

(b)

Figure 12. (a) Energy loss comparison among four architectures for one-day operation (b) energy loss comparison between CGCSA and DGDSA with various power-sharing levels.

It can be concluded from Figure 12a that, for a village with lower usage diversity, DGDSA having generation close to the utilization experiences minimal distribution losses, and therefore overall energy losses are very low compared to all other architectures. Similarly, from Figure 12b, it may be concluded that, for lower levels of power-sharing, DGDSA is not feasible, as it incurs higher conversion losses. However, for a moderate level of power-sharing i.e., up to 40\%, DGDSA experiences 13\% fewer energy losses compared to CGCSA in a full-day operation scenario. Thus, for DGDSA, power-sharing levels can be optimized to achieve minimal energy losses.

\subsection{PV and Battery Sizing Comparison}

In an islanded solar PV DC microgrid, where the grid connection is not available, the only source of generation is through PV modules, therefore, system losses directly impact the required PV sizing for the fulfillment of load demand. Generally, higher system losses result in higher PV size requirements. Moreover, since solar PV is intermittent in nature, for non-sunlight hours, the battery supplies the load demand and also fulfills the system losses; therefore, battery sizing also depends upon the energy losses incurred in the path of power flow. As demonstrated in Section 3, the distribution losses depend upon various system parameters, including distribution voltage level, amount of power to be distributed, and the choice of the distribution conductor. Using the case study parameters and the framework for sizing calculation presented in the above section, a comparison among four architectures for PV sizing (left y-axis) and battery sizing (right y-axis) requirements is shown in Figure 13. 


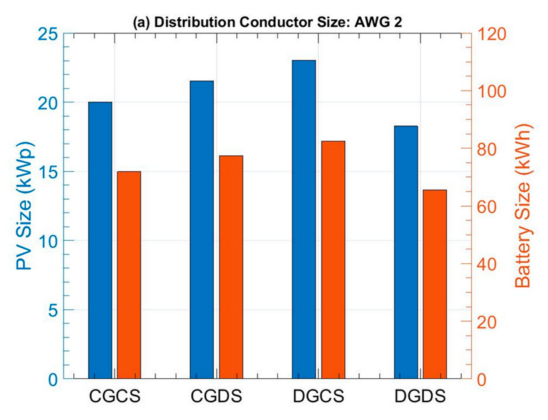

(a)

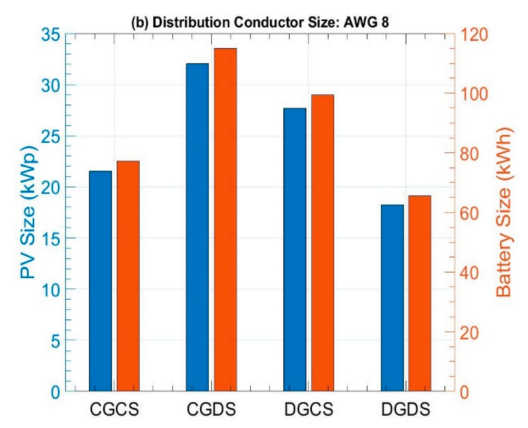

(b)

Figure 13. (a) PV sizing (left y-axis) and battery sizing (right y-axis) comparison among four architectures with AWG 2 conductor (b) PV sizing (left y-axis) and battery sizing (right y-axis) comparison among four architectures with AWG 8 conductor.

From Figure 13a,b, it can be seen that the higher the system losses are, the more sizing requirements there are. For instance, owing to minimal distribution and conversion losses, battery and PV sizing for DGDSA and CGCSA is lower as compared to CGDSA, and DGCSA. Thus, not only from the system losses perspective (as demonstrated in Figures 10-12) but also from sizing, DGDSA and CGCSA outperform CGDSA and DGCSA. Moreover, from the comparison of Figure 13a,b, using American Wire Guage (AWG) system, it can be observed that using a thin distribution conductor with higher resistance (AWG 8) in comparison to a thick conductor with relatively lower resistance (AWG 2) results in higher distribution losses, which can be directly translated in higher PV and battery sizing requirements. However, a thick conductor requires a higher initial up-front cost, and optimization among the upfront cost and the operational cost (in terms of distribution losses) can be made for better resource utilization. The analysis shown here considers only two gauge levels of distribution conduction thickness, however, the proposed framework can be employed for other conductor sizes as well.

As discussed earlier, the usage diversity and the associated power-sharing among the neighboring households result in a distribution loss in DGDSA. This distribution loss directly affects the system sizing, as demonstrated by Figure 14. Using the case study parameters and applying the proposed system sizing framework, PV and battery sizing for the various percentage of power-sharing in DGDSA is determined and plotted against the sizing of the most commonly employed CGCSA.

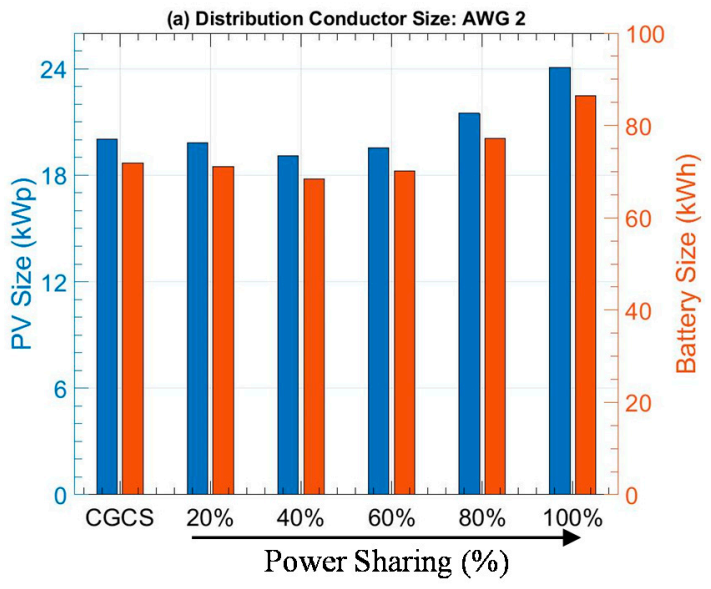

(a)

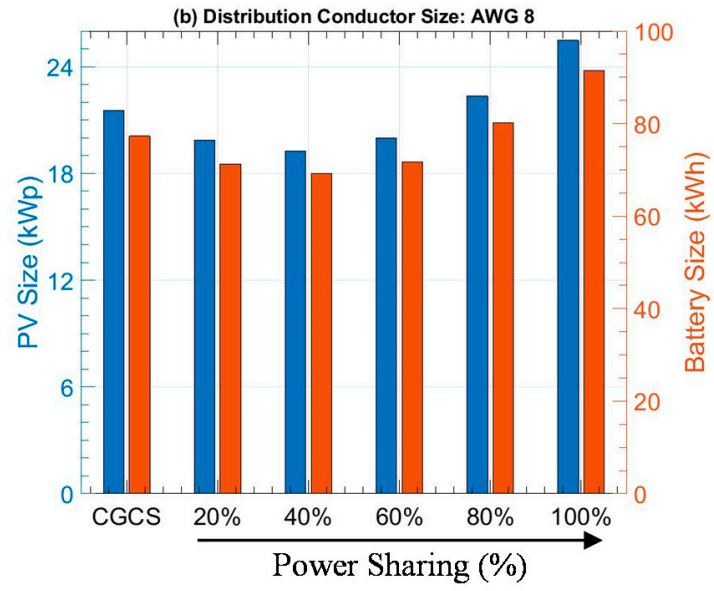

(b)

Figure 14. (a) PV and battery sizing comparison between CGCSA and DGDSA with various power-sharing levels with AWG 2 conductor (b) time-varying distribution losses of CGCSA vs. DGDSA at variable power-sharing with AWG 8 conductor. 
It can be verified from Figure 14 that, with very high and very low levels of power-sharing in DGDSA, system sizing requirements are higher as compared to CGCSA. For instance, Figure 14a,b system sizing (PV and battery sizing) requirements at $40 \%$ of power-sharing are lower than both CGCSA and $20 \%$ of power-sharing in DGDSA. Moreover, system sizing requirements significantly reduce with the usage of a thick conductor of higher AWG. Therefore, due to the nonlinear behavior of power-sharing percentage, system losses, and associated PV and battery sizing requirements, the proposed framework can be used for comparative analysis, and system sizing. Based on the comparative analysis framework, a particular architecture can be selected for optimal resource utilization in future rural electrification deployments.

\section{Conclusions}

A detailed comparative analysis of various PV-based DC microgrid architectures for rural electrification applications is presented in this work. Along with the details of architecture, a mathematical framework for the evaluation of losses and system sizing is also presented. Various parameters that affect system losses and sizing are identified, and architecture's efficiencies are evaluated accordingly. In all operation scenarios, DGDSA and CGCSA outperform the other two architectures, i.e., CGDSA and DGCSA. Although CGCSA is simple to control and implement, it has been shown that, for villages with lower usage diversity and minimal power-sharing requirements, DGDSA can be the optimal choice concerning system losses and system sizing. However, with the increase in usage diversity and power-sharing requirements, not only do the control and the complexity enhance but the system losses and sizing requirements enhance as well. In such scenarios, CGCSA can be the optimal choice for system designers. Different power-sharing levels can be evaluated using the proposed framework; therefore, optimal resource utilization can be achieved. Moreover, DGDSA offers enhanced functionalities, including the capability of bidirectional energy transaction and power pooling for community applications. Therefore, it is the choice of the designer and the specifications of the subscriber as to which architecture is preferred, however, the framework presented is generic in nature and gives complete insight into loss analysis and sizing calculation for all possible architectures. The proposed model can be used by system planners for efficiency analysis, system sizing, and architecture selection. Moreover, the proposed model and the associated analysis can be extended to evaluate the additional losses, e.g., partial shading effects and battery charging/discharging losses along with their dependence on the architecture under consideration. The proposed sizing framework can also be extended for upfront cost analysis, and results may be validated using standard renewable energy planning software, e.g., Hybrid Optimization Model for Multiple Energy Resources (HOMER) in future work. Researchers can also extend this model for developing an optimal peer-to-peer power-sharing framework in rural microgrids. The introduction of proper-sized centralized storage could improve performances of the DGDSA by mitigating the complexity of the system control and by optimizing the power-sharing requirements. Therefore, this work may also pave ways for the development of hybrid architectures, e.g., distributed generation architecture with mixed (distributed and centralized) storage for efficient rural electrification implementations in the future.

Author Contributions: Conceptualization, M.N.; methodology, M.N., S.I.; software, M.N., S.I.; validation, M.N., S.I.; formal analysis, M.N., H.A.K., J.C.V., J.M.G.; writing-original draft preparation, M.N.; writing-review and editing, J.C.V., J.M.G., H.A.K.; supervision, J.C.V., J.M.G., H.A.K.; project administration, J.C.V., J.M.G.; funding acquisition, J.C.V., J.M.G. All authors have read and agreed to the published version of the manuscript.

Funding: This work was funded by a "Villum Investigator grant" (no. 25920) from The Villum Fonden, Denmark.

Conflicts of Interest: The authors declare no conflict of interest. 


\section{Acronyms}

$\begin{array}{ll}\text { DG } & \text { Distributed generation } \\ \text { PV } & \text { Photovoltaic } \\ \text { MGP } & \text { Mera Gao Power } \\ \text { UP } & \text { Uttar Pradesh } \\ \text { TERI } & \text { The Energy and Resource Institute } \\ \text { WWF } & \text { Worldwide Fund } \\ \text { JEEP } & \text { Joint energy and environment project } \\ \text { CGCSA } & \text { Central generation and central storage architecture } \\ \text { CGDSA } & \text { Central generation and distributed storage architecture } \\ \text { DGCSA } & \text { Distributed generation and central storage architecture } \\ \text { DGDSA } & \text { Distributed generation and distributed storage architecture } \\ \text { MPPT } & \text { Maximum power point tracking } \\ \text { NR } & \text { Newton-Raphson } \\ \text { SOC } & \text { State of charge } \\ \text { PSH } & \text { Peak sunlight hour } \\ \text { AWG } & \text { American Wire Guage } \\ \text { HOMER } & \text { Hybrid Optimization Model for Multiple Energy Resources }\end{array}$

\section{References}

1. Nasir, M.; Khan, H.A.; Hussain, A.; Mateen, L.; Zaffar, N.A. Solar PV-Based Scalable DC Microgrid for Rural Electrification in Developing Regions. IEEE Trans. Sustain. Energy 2017, 9, 390-399. [CrossRef]

2. Peters, J.; Sievert, M. Impacts of Rural Electrification Revisited: The African Context. Revue d'Econom. Développ. 2015, 23, 77. [CrossRef]

3. Nasir, M.; Khan, H.A.; Zaffar, N.A.; Vasquez, J.C.; Guerrero, J.M. Scalable Solar dc Micrigrids: On the Path to Revolutionizing the Electrification Architecture of Developing Communities. IEEE Electr. Mag. 2018, 6, 63-72. [CrossRef]

4. Reiche, K.; Covarrubias, A.; Martinot, E. Expanding electricity access to remote areas: Off-grid rural electrification in developing countries. Fuel 2000, 1, 1-4.

5. IEA. World Energy Outlook 2020; IEA: Paris, France, 2020; Available online: https://www.iea.org/reports/ world-energy-outlook (accessed on 4 November 2020).

6. Lam, N.L.; Smith, K.R.; Gauthier, A.; Bates, M.N. Kerosene: A Review of Household Uses and their Hazards in Low- and Middle-Income Countries. J. Toxicol. Environ. Health Part B 2012, 15, 396-432. [CrossRef] [PubMed]

7. Guerrero, J.M.; Blaabjerg, F.; Zhelev, T.; Hemmes, K.; Monmasson, E.; Jemei, S.; Comech, M.P.; Granadino, R.; Frau, J.I. Distributed Generation: Toward a New Energy Paradigm. IEEE Ind. Electron. Mag. 2010, 4, 52-64. [CrossRef]

8. Soshinskaya, M.; Crijns-Graus, W.H.; Guerrero, J.M.; Vasquez, J.C. Microgrids: Experiences, barriers and success factors. Renew. Sustain. Energy Rev. 2014, 40, 659-672. [CrossRef]

9. Hirsch, A.; Parag, Y.; Guerrero, J. Microgrids: A review of technologies, key drivers, and outstanding issues. Renew. Sustain. Energy Rev. 2018, 90, 402-411. [CrossRef]

10. Palit, D.; Malhotra, S. Energizing rural India using micro grids: The case of solar DC micro-grids in Uttar Pradesh State, India. In In Proceedings of the Third International Conference: Micro Perspectives for Decentralized Energy Supply, Bangalore, India, 23-25 April 2015.

11. Justo, J.J.; Mwasilu, F.; Lee, J.; Jung, J.-W. AC-microgrids versus DC-microgrids with distributed energy resources: A review. Renew. Sustain. Energy Rev. 2013, 24, 387-405. [CrossRef]

12. Gelani, H.E.; Dastgeer, F.; Siraj, K.; Nasir, M.; Niazi, K.A.K.; Yang, Y. Efficiency Comparison of AC and DC Distribution Networks for Modern Residential Localities. Appl. Sci. 2019, 9, 582. [CrossRef]

13. Balls, J.N.; Fischer, H.W. Electricity-Centered Clientelism and the Contradictions of Private Solar Microgrids in India. Ann. Am. Assoc. Geogr. 2019, 109, 465-475. [CrossRef]

14. Yadav, P.; Davies, P.J.; Palit, D. Distributed solar photovoltaics landscape in Uttar Pradesh, India: Lessons for transition to decentralised rural electrification. Energy Strategy Rev. 2019, 26, 100392. [CrossRef] 
15. Palit, D. Solar energy programs for rural electrification: Experiences and lessons from South Asia. Energy Sustain. Dev. 2013, 17, 270-279. [CrossRef]

16. Chowdhury, S.A.; Kirchoff, S.G.H.; Magnasco, A.; Hasan, M. Community Based Decentralized DC Nanogrids for Combined Household and Productive Use, in IDCOL RED Initiative under Renewable Energy and Energy Efficiency Projects; Centre for Energy Research, United in Ternational University: Dhaka, Bangladesh, 2016; p. 1209.

17. Ainah, P.K.; Folly, K.A. Development of Micro-Grid in Sub-Saharan Africa: An Overview. Int. Rev. Electr. Eng. 2015, 10, 633. [CrossRef]

18. AbdelGayed, T.S.; Morsi, W.G.; Sidhu, T.S. Fault Detection and Classification Based on Co-training of Semisupervised Machine Learning. IEEE Trans. Ind. Electron. 2017, 65, 1595-1605. [CrossRef]

19. Madduri, P.A.; Poon, J.; Rosa, J.; Podolsky, M.; Brewer, E.; Sanders, S.R. Scalable DC Microgrids for Rural Electrification in Emerging Regions. IEEE J. Emerg. Sel. Top. Power Electron. 2016, 4, 1195-1205. [CrossRef]

20. Nasir, M.; Khan, H.A.; Niazi, K.A.K.; Jin, Z.; Guerrero, J.M. Dual-loop control strategy applied to PV/battery-based islanded DC microgrids for swarm electrification of developing regions. J. Eng. 2019, 2019, 5298-5302. [CrossRef]

21. Nasir, M.; Jin, Z.; Khan, H.A.; Zaffar, N.A.; Vasquez, J.C.; Guerrero, J.M. A Decentralized Control Architecture Applied to DC Nanogrid Clusters for Rural Electrification in Developing Regions. IEEE Trans. Power Electron. 2018, 34, 1773-1785. [CrossRef]

22. Nasir, M.; Anees, M.; Khan, H.A.; Guerrero, J.M. Dual-loop control strategy applied to the cluster of multiple nanogrids for rural electrification applications. IET Smart Grid 2019, 2, 327-335. [CrossRef]

23. Nasir, M.; Iqbal, S.; Khan, H.A. Optimal Planning and Design of Low-Voltage Low-Power Solar DC Microgrids. IEEE Trans. Power Syst. 2017, 33, 2919-2928. [CrossRef]

24. Hamza, M.; Shehroz, M.; Fazal, S.; Nasir, M.; Khan, H.A. Design and analysis of solar PV based low-power low-voltage DC microgrid architectures for rural electrification. In Proceedings of the IEEE Power \& Energy Society General Meeting, Chicago, IL, USA, 16-20 July 2017; Institute of Electrical and Electronics Engineers (IEEE): Piscataway, NJ, USA, 2018; pp. 1-5.

25. Iqbal, S.; Khan, H.A.; Nasir, M. Sizing of Low-Power DC Microgrids with Intermittent AC Grids in Developing Regions. Proceedings of the 2019 IEEE Power and Energy Society General Meeting, Atlanta, GA, USA, 4-8 August 2019; pp. 1-5. [CrossRef]

26. Nasir, M.; Anees, M.; Khan, H.A.; Khan, I.; Xu, Y.; Guerrero, J.M. Integration and Decentralized Control of Standalone Solar Home Systems for Off-Grid Community Applications. IEEE Trans. Ind. Appl. 2019, 55, 7240-7250. [CrossRef]

27. Nasir, M.; Zaffar, N.A.; Khan, H.A. Analysis on central and distributed architectures of solar powered DC microgrids. In Proceedings of the Clemson University Power Systems Conference (PSC), Clemson, SC, USA, 8-11 March 2016; Institute of Electrical and Electronics Engineers (IEEE): Piscataway, NJ, USA, 2016; pp. 1-6.

28. Kolar, J.W.; Krismer, F.; Lobsiger, Y.; Muhlethaler, J.; Nussbaumer, T.; Minibock, J. Extreme efficiency power electronics. In Proceedings of the 7th International Conference on Integrated Power Electronics Systems (CIPS), Nuremberg, Germany, 6-8 March 2012.

29. Nasir, M.; Khan, H.A. Solar photovoltaic integrated building scale hybrid AC/DC microgrid. In Proceedings of the 5th IET International Conference on Renewable Power Generation (RPG), London, UK, 21-23 September 2016; pp. 1-6.

30. Gelani, H.E.; Nasir, M.; Dastgeer, F.; Hussain, H. Efficiency comparison of alternating current (AC) and direct current (DC) distribution system at residential level with load characterization and daily load variation. Pak. Acad. Sci. 2017, 54, 111-118.

31. Khan, H.A.; Ahmad, H.F.; Nasir, M.; Nadeem, M.F.; Zaffar, N.A. Decentralised electric power delivery for rural electrification in Pakistan. Energy Policy 2018, 120, 312-323. [CrossRef]

32. Wunder, B.; Ott, L.; Kaiser, J.; Han, Y.; Fersterra, F.; Marz, M. Overview of different topologies and control strategies for DC micro grids. In Proceedings of the IEEE First International Conference on DC Microgrids (ICDCM), Atlanta, GA, USA, 7-10 June 2015; Institute of Electrical and Electronics Engineers (IEEE): Piscataway, NJ, USA, 2015; pp. 349-354.

33. Parra, D.; Swierczynski, M.; Stroe, D.I.; Norman, S.; Abdon, A.; Worlitschek, J.; O’Doherty, T.; Rodrigues, L.T.; Gillott, M.C.; Zhang, X.; et al. An interdisciplinary review of energy storage for communities: Challenges and perspectives. Renew. Sustain. Energy Rev. 2017, 79, 730-749. [CrossRef] 
34. Madduri, P.A.; Poon, J.; Rosa, J.; Podolsky, M.; Brewer, E.; Sanders, S. A scalable dc microgrid architecture for rural electrification in emerging regions. In Proceedings of the IEEE Applied Power Electronics Conference and Exposition (APEC), Charlotte, NC, USA, 15-19 March 2015; Institute of Electrical and Electronics Engineers (IEEE): Piscataway, NJ, USA, 2015; pp. 703-708.

35. Anand, S.; Rao, A.B. Models for Deployment of Solar PV Lighting Applications in Rural India. Energy Procedia 2016, 90, 455-462. [CrossRef]

36. Pervaiz, S.; Khan, H.A. Low irradiance loss quantification in c-Si panels for photovoltaic systems. J. Renew. Sustain. Energy 2015, 7, 013129. [CrossRef]

37. Maghami, M.R.; Hizam, H.; Gomes, C.; Radzi, M.A.; Rezadad, M.I.; Hajighorbani, S. Power loss due to soiling on solar panel: A review. Renew. Sustain. Energy Rev. 2016, 59, 1307-1316. [CrossRef]

38. Forniés, E.; Naranjo, F.; Mazo, M.; Ruiz, F. The influence of mismatch of solar cells on relative power loss of photovoltaic modules. Sol. Energy 2013, 97, 39-47. [CrossRef]

39. Solanki, C.S. Solar Photovoltaics: Fundamentals, Technologies and Applications, 3rd ed.; PHI Learning Private Learning: Delhi, India, 2015.

40. Bashir, N.; Sardar, H.S.; Nasir, M.; Hassan, N.U.; Khan, H.A. Lifetime maximization of lead-acid batteries in small scale UPS and distributed generation systems. In Proceedings of the IEEE Manchester PowerTech, Manchester, UK, 18-22 June 2017; Institute of Electrical and Electronics Engineers (IEEE): Piscataway, NJ, USA, 2017; pp. 1-6.

41. National Solar Radiation Database (NSRDB). National Renewable Energy Laboratory (NREL). Available online: https://nsrdb.nrel.gov/ (accessed on 4 November 2020).

42. Khan, R.; Schulz, N.N.; Nasir, M. Distribution Loss Analysis of DC Microgrids for Rural Electrification. In Proceedings of the IEEE Global Humanitarian Technology Conference (GHTC), Seattle, WA, USA, 17-22 October 2019; Institute of Electrical and Electronics Engineers (IEEE): Piscataway, NJ, USA, 2019.

Publisher's Note: MDPI stays neutral with regard to jurisdictional claims in published maps and institutional affiliations.

(C) 2020 by the authors. Licensee MDPI, Basel, Switzerland. This article is an open access article distributed under the terms and conditions of the Creative Commons Attribution (CC BY) license (http://creativecommons.org/licenses/by/4.0/). 